\title{
Design of Double-rows Sugarcane Harvester Control System Based on PLC
}

\author{
Liping $\mathrm{Xu}^{1, \mathrm{a}}$ and Liujin $\mathrm{Cai}^{1, \mathrm{~b}^{*}}$ \\ ${ }^{1}$ School of Mechatronic Engineering, Henan University of Science and Technology, Luoyang, \\ 471003, China \\ aemail:xlpzz@163.com, bemail:yycailj@163.com \\ *The corresponding author
}

Keywords: Sugarcane harvester; Control system; PLC; Touch screen; Monitor

\begin{abstract}
The double-rows sugarcane harvester is a large agricultural machinery, it has complicated working conditions and high failure rate in the process of harvesting, which seriously affect the quality and efficiency of harvesting. Aiming at these problems, the electrical and hydraulic control system using PLC as the control core was designed. Through the PC monitoring program, the system could complete the online settings and monitor the operation conditions of the control parameters to achieve complex logic control and matching control of each actuator's movement speed. The results from the harvesting test in Guangxi farm show that the control system has advantages of convenient and reliable operation, reducing the failure rate, and improving the quality and efficiency of harvesting.
\end{abstract}

\section{Introduction}

The double-rows sugarcane harvester is a new type of high power harvester derived based on single type sugarcane harvester. The biggest difference is the double-rows sugarcane harvester has two similar machine assemblies, sugarcane cutter group, picking up channel assembly and a larger leaf stripping channel assembly. With greater engine power, greater channel logistics capabilities, greater sugarcane collection hopper, faster walking and better driving experience. As shown in fig. 1. Harvesting system conditions are complex, with multiple actuators, linkage and interlock action, complicated operation, so the development of a set of PLC control system, increase the degree of automation, reduce energy consumption, improve the quality and efficiency of harvesting [1-3].

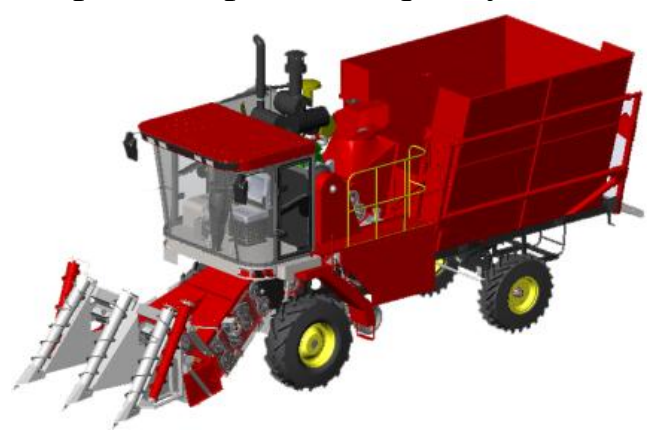

Figure 1. The double-rows sugarcane harvester

\section{Sugarcane Harvester PLC Control System}

Sugarcane harvester control system is mainly for the engine, hydraulic pump, hydraulic valve (conventional directional valve and proportional direction valve) and actuator (hydraulic cylinder, hydraulic motor) monitoring of hydraulic oil temperature, pressure, speed, switch and other parameters. The hydraulic system and the working state of the harvester are monitored and protected by pressure, rotating speed and temperature sensor, buzzer and fuse. In order to make the system stable and reliable operation and control system using PLC as the control core, the input data related to PLC sensor, integrated operation signal measurement PLC for various sensor value 
and setting value and the driver, a control signal to control the engine, hydraulic pump, hydraulic control valves and other machine. The basic structure of the electronic control system is shown in fig 2.

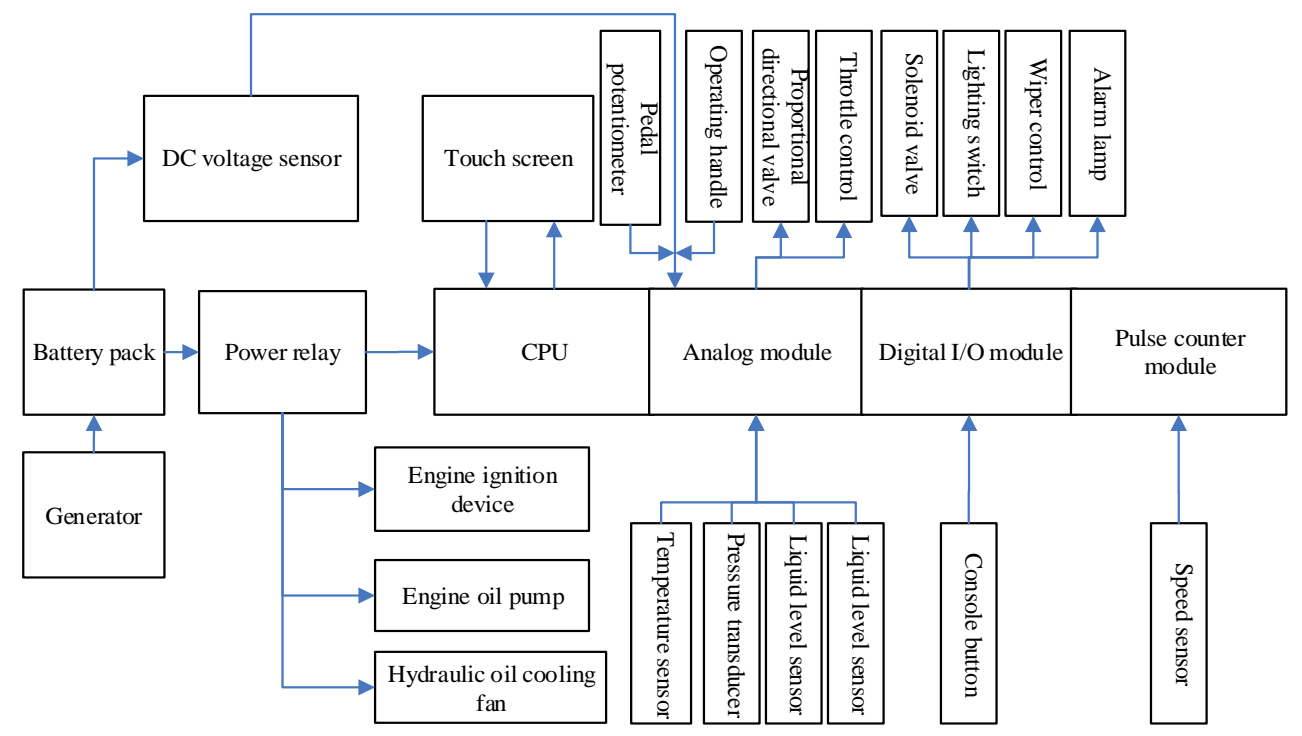

Figure 2. Electronic control system of harvester

\section{Hardware Composition of PLC Control System}

PLC model should meet the control objectives and the number of sensors required to detect the number of interfaces, as far as possible to choose high stability, cost-effective models and modules. Considering the cost, function and data interface of PLC, the CPU 313C-2 DP in SIEMENS PLC S7-300 series is chosen as the control core [4-6]. According to the control object and the type of the sensor, the control object, the control method and the signal type of the sugarcane harvester electrical control system are summarized, which are shown in Table 1 and table 2.

Table 1 Harvester control function

\begin{tabular}{cccc}
\hline Function & Control input & Control object & Signal type \\
\hline Forward / backward & Electric control handle & Walking pump proportional valve & Analog quantity \\
Walking speed & Pedal & Engine Throttle & Analog quantity \\
Vehicle steering & Electric control handle & Steering solenoid directional valve & Switch quantity \\
Hopper lift & Console button & Electromagnetic direction valve for & Switch quantity \\
Cutter and sugarcane & Console button & Lifting hydraulic cylinder solenoid & Switch quantity \\
lifting & Operation panel & directional valve & Analog quantity \\
Cutter speed & Operation panel & Proportional pump and motor & Analog quantity \\
Channel speed & Console button & Solenoid directional valve & Switch quantity \\
Sugarcane rotation & & &
\end{tabular}


Table 2 Harvester test item

\begin{tabular}{ccccc}
\hline & Detection object & Sensor type & Detection method & Signal type \\
\cline { 2 - 5 } & Hydraulic motor speed & Hall sensor & PLC counter module & Switch quantity \\
Engine speed & Hall sensor & PLC counter module & Switch quantity \\
Signal & Motor pressure & Pressure sensor & PLC analog module & Analog quantity \\
& Cylinder pressure & Pressure sensor & PLC analog module & Analog quantity \\
& Battery voltage & Voltage sensor & PLC analog module & Analog quantity \\
& Tank temperature & Temperature sensor & PLC analog module & Analog quantity \\
& Oil temperature sensor & Temperature sensor & PLC analog module & Analog quantity \\
& Fuel tank level & Liquid level sensor & PLC analog module & Analog quantity \\
\hline
\end{tabular}

Table 1 and Table 2 show that the PLC data interface requires 10 digital output, the CPU 313C-2 DP model PLC has a built-in digital I/O interface 16, so it is no need to select a digital module. Proportional valve and pressure sensor is used in analog PLC have 6 analog output interface and 6 analog input interface, so it needs to choose two of 4 input and two output 4 analog control module SM323. There are 8 institutions need to measure the speed, the use of magnetic resistance sensor to measure the speed of rotation, you can use the PLC counter module to measure the number of pulses to calculate the speed. So choose a PLC 8 channel counter module FM350-2. Taking into account the need for the upgrading of electrical and hydraulic systems, a certain amount of standby interface is reserved when selecting the interface module [6-7].

\section{Software Design of PLC Control System}

SIEMENS PLC programming includes the hardware configuration and control program written in two parts. According to the actual use of sugarcane harvester and the requirements of the operation of the process, the main electrical control system flow chart, and software programming. The electrical control flow chart is shown in fig.3.

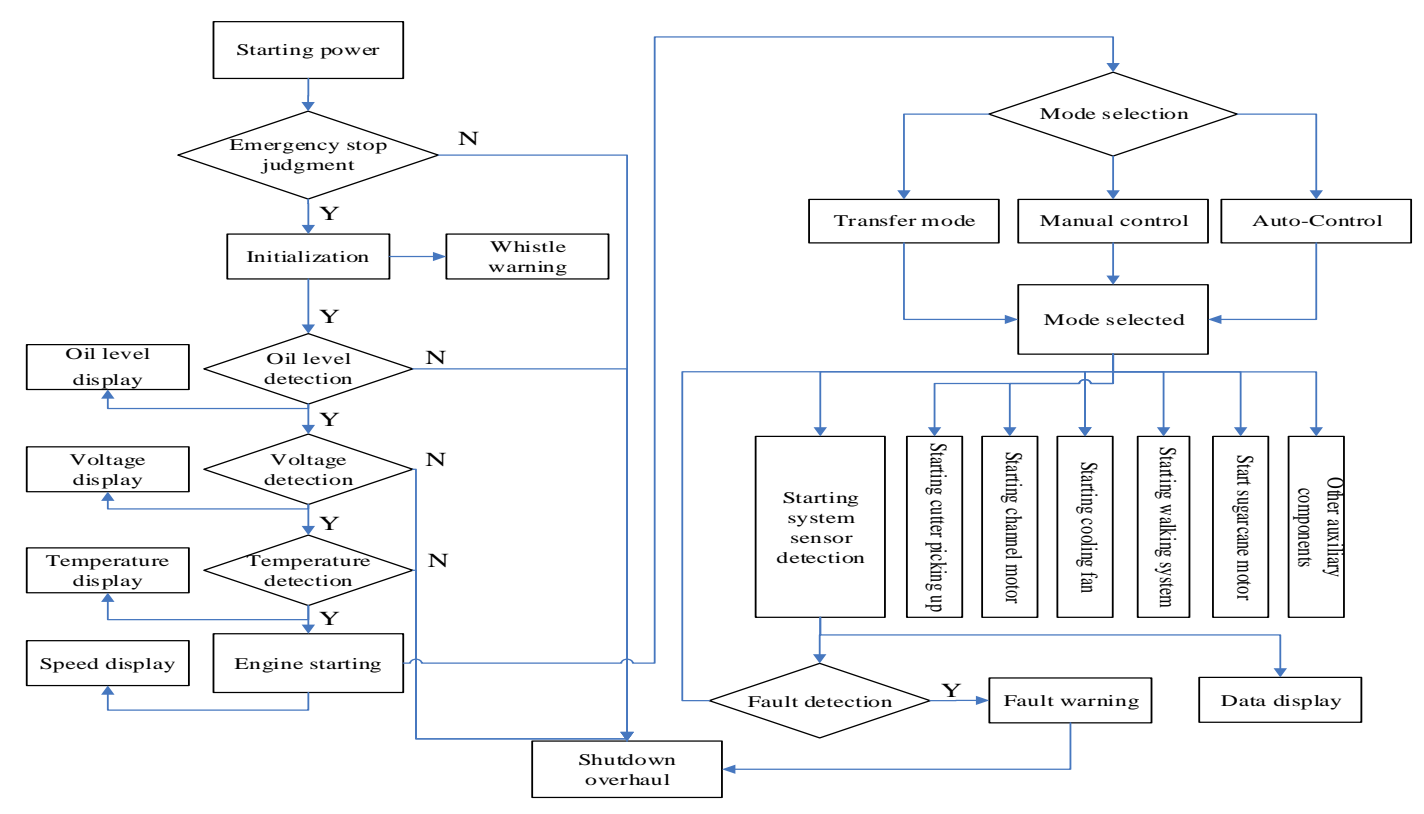

Figure 3. Main flow chart of electrical system control 


\section{Touch Screen Monitor Interface Design}

Good touch screen monitoring system allows operators to better understand the operation of the machine, improve work efficiency, reduce labor intensity.

Structure Design of Touch Screen Monitoring System. The monitoring system of sugarcane combine is shown in Figure 4. The monitoring system is mainly composed of touch screen, various types of sensors, PLC and related peripherals. Speed measurement by Holzer sensor is used to collect the motor and the speed of the engine speed signal after adjusting circuit, are calculated by the high speed counter of PLC; pressure sensor, voltage sensor and temperature sensor can output 4-20mA standard signal, analog acquisition module can be directly detected by PLC signal. The data exchange between the touch screen and PLC through serial communication, the touch screen can display the important parameters of PLC in real time, and judge the running state of the harvester [8].

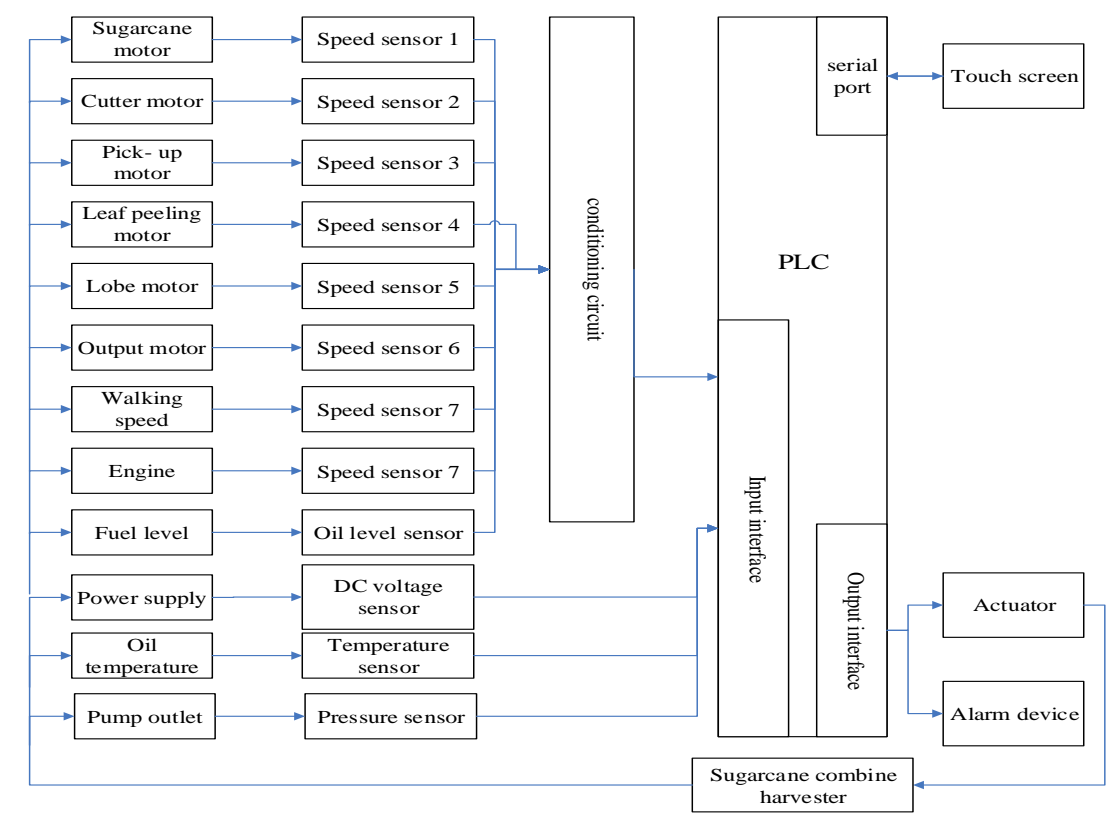

Figure 4. The structure of sugarcane harvester monitoring system

Interface Design of Touch Screen Monitoring System. Considering the problems of environment and cost, use YKHMI configuration software to optimize and control the Shenzhen summit as the monitoring platform, touch screen interface editor of sugarcane harvester monitoring system. S1001A model of touch screen, touch screen with $800 \mathrm{MHz}$ A8 processor, 128M FLASH storage space, the panel can be waterproof, dustproof, shockproof, strong anti-interference ability. Sugarcane harvester touch screen monitoring system for the main interface, in the configuration, the control variable and the PLC interface of the button in the dashboard, and sensor data connectivity, when the control variables of PLC changes, the color of the button will change. When the system is activated, the button will display the green, and start the relevant functions of the subroutine. Change of oil temperature and fuel level sensor when the relevant data will lead to a change in the interface relative to the corresponding occurrence histogram. When an emergency occurs in the system, and the mechanical emergency stop button mechanical failure occurs, you can stop the operation of the through the touch screen [9].

For the parameter setting interface, set up three working mode, a transition mode, manual mode and automatic control mode, and the parameters of their custom input box, the user can according to the harvest experience, set the main parameters of the touch screen, the system will automatically be batch parameter assignment to the PLC program, implementation of the relevant operation.

The harvester monitoring system also set up a fault diagnosis and alarm history table, fault alarm function is the use of sensor data to realize the comprehensive judgment of running state of the sugarcane harvester is monitored, once the abnormal fault can send out sound and light alarm to 
remind the operator early troubleshooting problems, reduce maintenance time and reduce maintenance costs. Not only improve the working environment, but also improve the operation efficiency[10].

\section{Summary}

The control system for the field test in Guangxi harvest farm, increase the degree of automation, to achieve complex logic control of the executive mechanism of harvesters, operation, friendly man-machine interface makes the operator can real-time understanding of the system has the advantages of simple operation, high reliability, easy to modify parameters to improve the harvest, the harvest quality and efficiency.

\section{Acknowledgements}

The authors gratefully acknowledge natural science foundation of henan university of science and technology for financial support of this research work.(2015QN017)

\section{References}

[1] J. Hou: Study on the Competitiveness of Sugarcane Industry in Guangxi Autonomous Region (MS., Guangxi University, China 2012), p.12 (In Chinese).

[2] G. N. Han, Z. L. Xie, X. Y. Liang: Aeronautical Computing Technique, Vol. 40 (2010) No.5, p.122 (In Chinese).

[3] M. Xu, Y. Zhang: Journal of Agricultural Mechanization Research, (2014) No.11, p.36 (In Chinese).

[4] F. L. Wang: Digital Emulation on Fluid Drive System of Sugarcane Mill \& PLC Integrated Control on the Productive Line for Grinding Cane in Sugar-refinery(MS., Guangxi university, China 2012), p.42 (In Chinese).

[5] J. Chen, F. L. Wang, K. M. Liu: Machine Tool \& Hydraulic, (2005) No.3, p.54 (In Chinese).

[6] W. Guo, L. L. Tian, Y. L. Zhang: Power System Protection and Control, (2014) No.16, p.163 (In Chinese).

[7] J. Zhu: Construction Machinery Today, (2013) No.11, p.16 (In Chinese).

[8] X. F. Jiang, W. F. Shi, Y. J. Liu: Electric Power Automation Equipment, Vol. 31 (2011) No.1, p.145 (In Chinese).

[9] P. Lu: process automation instrumentation, Vol. 31 (2010) No.8, p.102 (In Chinese).

[10] J. Wang, D. H. Zhang, J. P. Li: Journal of Northeastern University (Natural Science), Vol. 22 (2001) No.4, p.58 (In Chinese). 